\title{
The effect of amplitude envelopes of each amplitude modulated wave on the timbre of compound tones consisting of three amplitude modulated waves
}

\author{
Shin-ichiro Iwamiya \\ Department of Acoustic Design, Kyushu Institute of Design, \\ 4-9-1, Shiobaru, Minami-ku, Fukuoka, 815 Japan
}

(Received 18 March 1994)

\begin{abstract}
The timbre of compound tones consisting of three amplitude modulated waves is examined by psychoacoustical experiments using a similarity judgement. The obtained data are analyzed by a multi-dimensional scaling (MDS) method. The MDS solutions are interpreted by the regression equations as a function of the envelope correlation coefficients between the AM waves. In particular, the envelope correlations between adjacent AM waves are the determinant factor. When the modulation rate is slow enough, the fine structure of amplitude envelopes of each AM wave affects the timbre. However, the modulation rate is faster than the auditory temporal resolving power, the fine structure has no effect and the timbre is determined by only the envelope correlations. The auditory system has a function to detect the amplitude envelope correlation between the output signals of the different auditory filters. The present study shows how this function contributes to timbre perception.
\end{abstract}

Keywords: Timbre, Vibrato, Amplitude modulation, Multi-dimensional scaling (MDS) method, Amplitude envelope correlation

PACS number: 43. 66. Jh

\section{INTRODUCTION}

Timbre is one of the most important three auditory qualities; the other two are pitch and loudness. Timbre is defined by the acoustical spectrum, the attack pattern, and the decay pattern of sound. Furthermore, the amplitude envelope of the steady portion is an important physical property to define timbre.

Actual musical sounds consist of many harmonics (sometimes they are nearly harmonic) and the harmonics have their own amplitude envelope patterns. In the case of vibrato sounds of violin the amplitude of each harmonic is modulated according to the resonance characteristics of the body of the violin (Fletcher and Sanders, 1967). Frequency modulations of each harmonic of the vibrato sound are in phase, but the phase of the amplitude modulation of each harmonic depends on the slope of the resonance curve of the corresponding frequency region. When the slope is positive, FM and AM are in phase (frequency and amplitude simultaneously increase and decrease), and when the slope is negative, FM and AM are out of phase (when frequency increases amplitude decreases and vice versa). ("AM" stands for "amplitude modulation" or "amplitude modulated," and "FM" stands for "frequency modulation" or "frequency modulated.") Then the amplitude modulation of each harmonic has a different phase. Some of them are in phase and the others are out of phase. As a result, the vibrato of violin causes periodic variation of spectrum structure.

Fletcher and Sanders (1967) tried to synthesize violin vibrato tones by introducing these spectrum structure changes. The phase relationship among AMs of each harmonic of the synthesized tones was adjusted to be the same as that of real tones. In 
this way, they could successfully obtain the quality of violin vibrato tones. For novices, the synthetic tones were satisfactory duplications of the real tones although the experts could detect the difference by very careful listening.

Similar phenomenon was found in vocal vibrato (Sundberg, 1982). According to the resonance of vocal tract (formant), vocal vibrato tones accompany amplitude modulations of each harmonic. Sundberg suggests that these amplitude modulations may give information about the frequency locations of the formants and facilitate vowel identification for high-pitched vowels. Furthermore, many electronic instruments and sound effectors (for example, "Phase shifter" and "Flanger") have similar functions which periodically change the temporal spectrum structure. For both natural and electronic musical instruments, different amplitude envelopes of each harmonic and the resulting periodic variation of spectrum structure is considered to contribute to the timbre of musical sounds.

However, there has not been systematic study on this issue from psychoacoustical point of view. The effects of phases among AMs on timbre have not been studied extensively. It is not clear how each amplitude envelope affects the timbre of musical sound. The purpose of this study is to clarify the determinant factor of timbre of compound tones consisting of $\mathrm{AM}$ waves as a function of phase differences among AMs. In this study some psychoacoustical experiments were conducted to investigate how the envelopes of each $\mathrm{AM}$ wave affect the timbre of sound.

In this study the compound tones consisting of three AM waves were used as sound stimuli. Three AM waves provide the minimum experimental condition in which the effects of amplitude envelopes of adjacent $A M$ waves, those of not-adjacent $\mathrm{AM}$ waves, and the relationship among these effects can be examined. These issues are considered to be important to investigate how the envelopes of each $A M$ wave affect the timbre of sound. They cannot be examined by two AM waves.

\section{EXPERIMENTAL METHOD}

The sound stimuli of the present experiment are defined as;

$$
\sum_{i=1}^{3}\left[1+\cos \left(2 \pi f_{\text {mod }} t+\theta_{i}\right)\right] \cos \left(2 \pi i f_{\mathrm{c}} t\right) .
$$

The carrier waves of each AM wave had the same amplitude and the modulation depth of each AM was $100 \%$. The fundamental frequency $\left(f_{\mathrm{c}}\right)$ of the carrier waves was $440 \mathrm{~Hz}$. The modulation wave was sinusoidal. The variation of amplitude envelope combination was due to the initial phase of each AM. The initial phase of AM was 0 degrees for the fundamental $\mathrm{AM}$ wave $\left(\theta_{1}=0\right.$ degrees), and $0,90,180$, and 270 degrees for the second and the third AM waves $\left(\theta_{2}, \theta_{3}=0,90,180\right.$, and 270 degrees). The duration of sound stimuli was $1.6 \mathrm{~s}$.

The experiments were done under two modulation frequency conditions. The modulation frequency $\left(f_{\text {mod }}\right)$ of one experiment was 4 and the other was $8 \mathrm{~Hz}$. The modulation frequency of vibrato sounds is between these values (Seashore, 1938).

The sound stimuli are defined by three digit numbers for the sake of convenience. Each number shows the initial phase of the modulation waves. The highest digit shows $\theta_{1}$ and the lowest digit shows $\theta_{3}$. The numbers $0,1,2$, and 3 mean $0,90,180$, and 270 degrees respectively. For example, "000" represents that $\theta_{1}=\theta_{2}=\theta_{3}=0$ degrees which means that all the amplitude modulations of each AM wave are in phase. "002" shows that $\theta_{1}=\theta_{2}=0$ degrees and $\theta_{3}=180$ degrees which means that the relation between the fundamental (or second) $\mathrm{AM}$ and the third AM is out of phase.

The sound stimuli were generated by a computer and presented diotically through headphones as pairs at a level of $70 \mathrm{~dB}$ SPL. The task of the subjects was to judge similarity of timbre on a 7-step scale between the pairs. All the combinations of the sixteen sound stimuli were presented. There was no pitch and loudness difference among the stimuli.

Five subjects with normal hearing, three males and two females, aged 20 to 24 years old, participated at $f_{\text {mod }}=8 \mathrm{~Hz}$. Four 'male and one female subjects with normal hearing, aged 21 to 24 years old, participated at $f_{\text {mod }}=4 \mathrm{~Hz}$. Three of them participated in both experiments.

The obtained similarity matrix of the sound stimuli was analyzed by a multi-dimensional scaling method, called INDSCAL (Arabie et al., 1987).

\section{RESULTS AND DISCUSSION}

The correlation coefficient between the scalar products derived from the original similarity matrix and those derived from the INDSCAL solution (coordinates in the MDS space) is taken as the 
criteria of INDSCAL solution (Arabie et al., 1987) for determination of the number of dimension. These correlations increase as the number of dimension increases and the three dimensional solutions show the saturation of increase of the correlations in both of the modulation frequency conditions. Then, the three dimensional solutions were chosen. The correlation coefficient is 0.82 at $f_{\text {mod }}=4 \mathrm{~Hz}$ and 0.83 at $f_{\text {mod }}=8 \mathrm{~Hz}$. The two dimensional solutions are same as the first two dimensions of the three dimensional solutions.

\subsection{When the Modulation Rate Is $8 \mathrm{~Hz}$}

Figure 1 shows the configuration of the sound

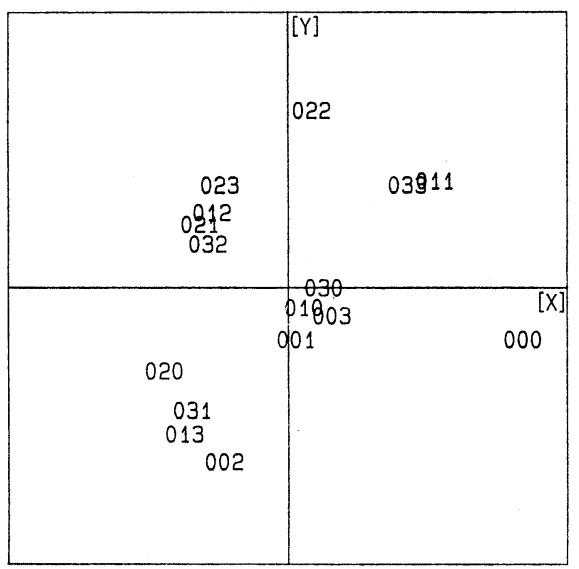

(a)

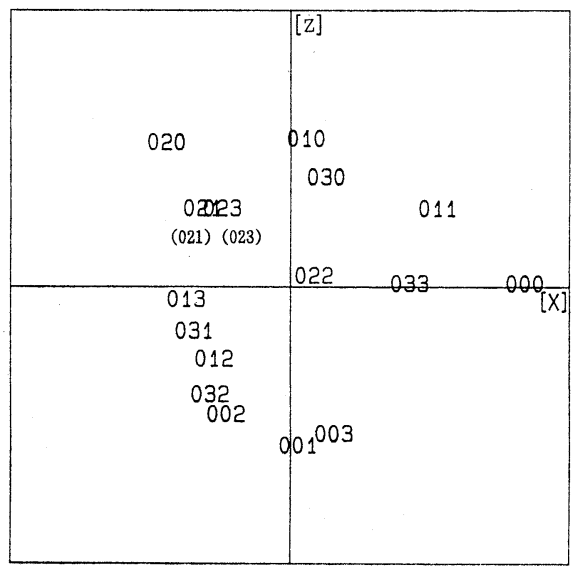

(b)

Fig. 1 Three dimensional INDSCAL solution when the modulation frequency is $8 \mathrm{~Hz}$. stimuli on the $x-y$ and $x-z$ planes at $f_{\mathrm{mod}}=8 \mathrm{~Hz}$. Figure 2 shows the individual weights of the subjects for each dimension at $f_{\text {mod }}=8 \mathrm{~Hz}$. If coordinates of stimuli or subjects overlap, the figures or the letters are shown in parentheses.

If the diagonal line is assumed from the third quadrant to the first quadrant on the $x-y$ plane, the sound stimuli are divided into three groups along the assumed line; they are $(002,031,013$, and 020), $(023,012,021,032,030,010,003$, and 001), and $(000,011,022$, and 033). This grouping is considered to be based on the phase difference between the second AM and the third AM. The distance is maximum between the stimuli with the phase dif-

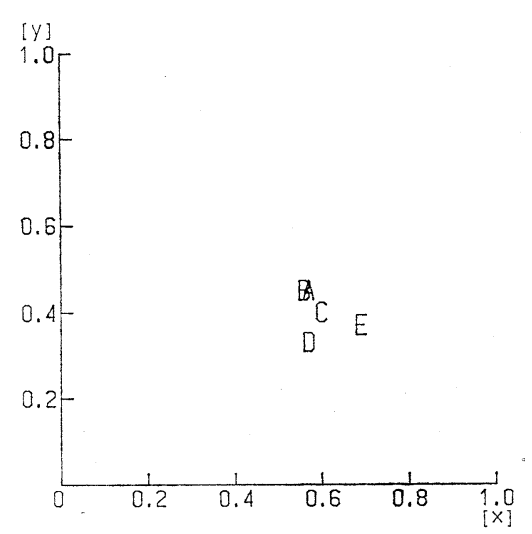

(a)

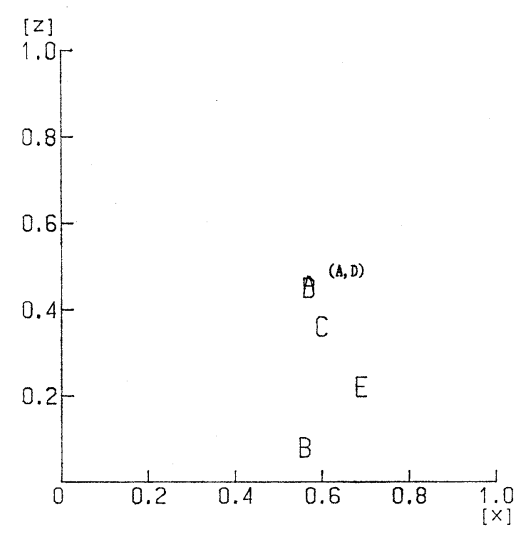

(b)

Fig. 2 Individual weights of the subjects for each dimension when the modulation frequency is $8 \mathrm{~Hz}$. Each letter shows a subject. 
ference of 0 and 180 degrees. Between them the stimuli with phase difference of 90 or 270 degrees are located.

From this relation we can assume that the amplitude envelope correlation between the second and the third $\mathrm{AM}$ waves is related to the timbre. If the modulation wave is sinusoidal, the correlation coefficient between the amplitude envelopes of the $i$-th and $j$-th AM waves $\left(\Phi_{i j}\right)$ is $\cos \left(\theta_{j}-\theta_{i}\right)$. So, the correlation coefficient between the envelopes of the second and the third AM waves $\left(\Phi_{23}\right)$ is -1 for $(002$, $031,013$, and 020$), 0$ for $(023,012,021,032,030$, 010,003 , and 001), and 1 for $(000,011,022$, and 033). In fact the stimuli are located according to the correlation coefficients between the envelopes of the second and the third AM waves along the assumed line from the third quadrant to the first quadrant.

Furthermore, orthogonal to this relation, we can see the order of the sound stimuli according to the correlation coefficients of the fundamental and the second envelopes from the forth quadrant to the second quadrant. For example, "033" and "011" $\left(\Phi_{12}=0\right)$ are middle of " $000 "\left(\Phi_{12}=1\right)$ and " 022 " $\left(\Phi_{12}=-1\right)$. This relation also shows that the timbre of compound tones consisting of three AM waves is related to the envelope correlation between the fundamental and the second AM waves.

In the $x-z$ plane we can see the effects of the envelope correlation of the fundamental and the third AM wave. Actually, the coordinate of $z$ axis is affected by the values of both $\Phi_{13}$ and $\Phi_{12}$. As $\Phi_{13}$ is larger and $\Phi_{12}$ is smaller, the coordinate of $z$-axis is more positive.

As a result, the configuration of the sound stimuli can be predicted by the linear multiple regression equations as a function of the envelope correlations between the AM waves;

$$
\begin{array}{ll}
x=0.15 \Phi_{12}+0.29 \Phi_{23} & (r=0.91), \\
y=-0.18 \Phi_{12}+0.27 \Phi_{23} & (r=0.92), \\
z=-0.24 \Phi_{12}+0.22 \Phi_{13} & (r=0.92),
\end{array}
$$

where $r$ is the multiple correlation coefficient.

Figure 2 shows that the weights for each axis generally decrease according to the order of $x, y$, and $z$. Furthermore, the subjects show similar weights for $x$ and $y$-axes. However, there are individual differences of the weights for the $z$-axis.

3.2 When the Modulation Rate Is $4 \mathrm{~Hz}$

Figure 3 shows the configuration of the sound

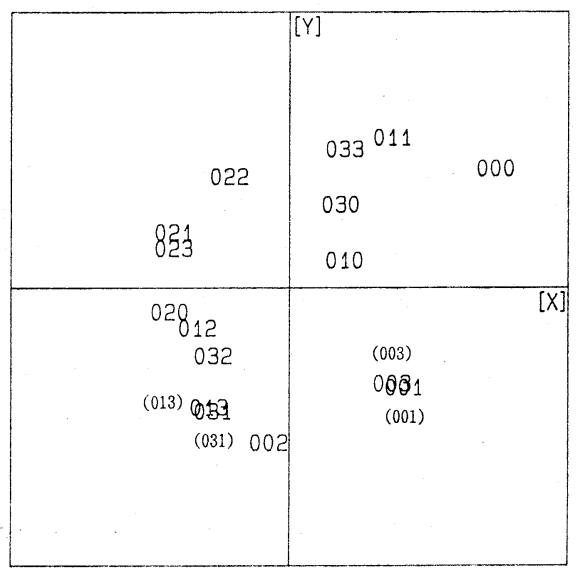

(a)

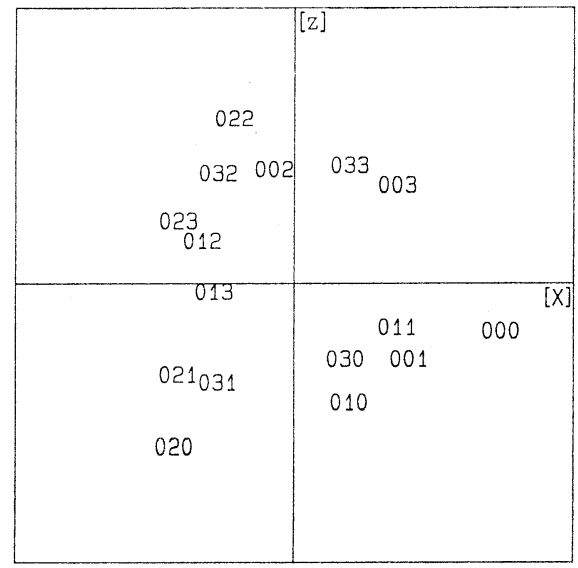

(b)

Fig. 3 Three dimensional INDSCAL solution when the modulation frequency is $4 \mathrm{~Hz}$.

stimuli on the $x-y$ and $x-z$ planes at $f_{\text {mod }}=4 \mathrm{~Hz}$. Figure 4 shows the individual weights of the subjects for each dimension at $f_{\text {mod }}=4 \mathrm{~Hz}$.

Similar to the results at $f_{\text {mod }}=8 \mathrm{~Hz}$, the configuration of the sound stimuli in the $x-y$ plane is related to the envelope correlation between the fundamental and the second AM waves, and that between the second and the third AM waves. The configuration of the sound stimuli on the $x-y$ plane can also be predicted by the multiple linear regression equations of the envelope correlation $\left(\Phi_{i j}\right)$;

$$
\begin{array}{ll}
x=0.26 \Phi_{12}+0.19 \Phi_{23} & (r=0.90), \\
y=-0.13 \Phi_{12}+0.23 \Phi_{23} & (r=0.91) .
\end{array}
$$




\section{S. IWAMIYA: THE EFFECT OF AMPLITUDE ENVELOPES ON TIMBRE}

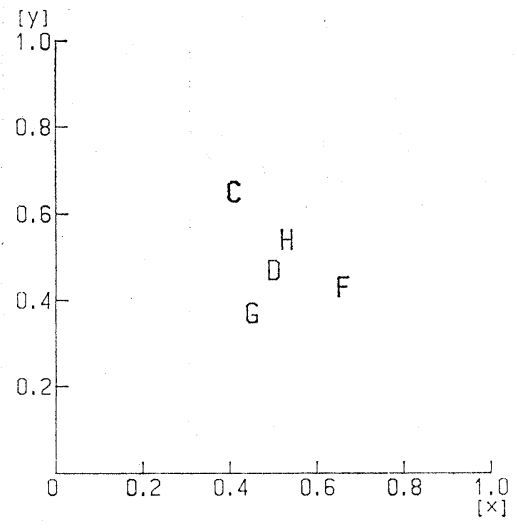

(a)

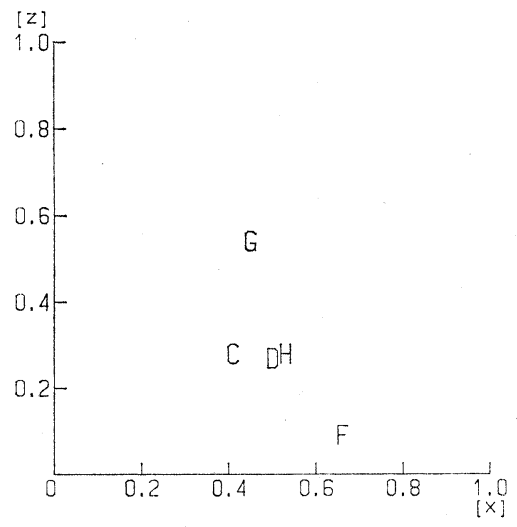

(b)

Fig. 4 Individual weights of the subjects for each dimension when the modulation frequency is $4 \mathrm{~Hz}$. Each letter shows a subject.

However, different tendency is shown along the $z$-axis. There are no clear differences in the location of the sound stimuli based on the phase differences of 90 and 270 degrees in Fig. 1 and in the $x-y$ plane of Fig. 3. However, along the $z$-axis of Fig. 3, differences are observed. For example, although the stimuli " 021 " and " 023 " are the middle of " 020 " and " 022 ," the distance between " 021 " and " 023 " may not be ignored. Then, the coordinate of $z$-axis at $f_{\text {mod }}=4 \mathrm{~Hz}$ cannot be predicted by the envelope correlation between the fundamental and the third AM waves because $\Phi_{13}$ is 0 for both " 021 " and "023." (The discriminability of the phase differences of 90 and 270 degrees is examined by the subsidiary experiment.)
Figure 4 shows a similar tendency to Fig. 2 although the weights for $x$ and $y$-axes are nearly the same in this case. The subjects show similar weights for $x$ and $y$-axis, and there are individual differences in the weights for the $z$-axis.

\section{SUBSIDIARY EXPERIMENT}

The subsidiary experiment was conducted to confirm that the discrimination of the phase differences of 90 and 270 degrees between amplitude modulations depends on the modulation frequency.

The discrimination experiment of sound stimuli " 021 " and " 023 " was done at $f_{\text {mod }}=2,4,8$, and 16 Hz. The sound pairs of " 021 \& 021,023 \& 023, $021 \& 023$, and $023 \& 021$ " were randomly presented in each modulation frequency condition. Each pair was presented 5 times. The task of the subjects was to judge whether the sounds in each pair were the same or different.

For comparison, a similar experiment was conducted for the sound stimuli " 020 " and "022" to examine the discriminability of the phase differences of 0 and 180 degrees.

Five subjects with normal hearing, three males and two females, aged 21 or 22 years old, participated. Four of them participated in either of the previous experiments.

Figure 5 shows the percentage of average correct answers under each condition. The difference of sound stimuli " 020 " and " 022 " is always discriminable if the 75-percent point is taken as a criterion. The difference of " 021 " and " 023 " is discriminable when $f_{\text {mod }}$ is lower than $4 \mathrm{~Hz}$, but it is not discrimina-

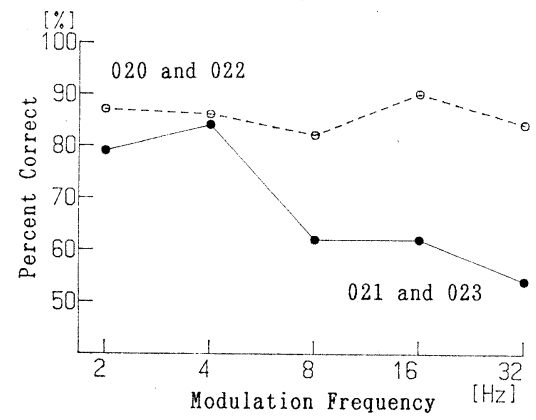

Fig. 5 Discrimination of the phase difference of 90 and 270 degrees between amplitude modulations, and that of 0 and 180 degrees as a function of the modulation frequency. 
ble when $f_{\text {mod }}$ is higher than $8 \mathrm{~Hz}$.

These results may reflect the temporal resolving power of the auditory system to the periodic amplitude modulation. When $f_{\text {mod }}$ is slower than 4 $\mathrm{Hz}$, the auditory system can follow each amplitude modulation respectively. Then, the auditory system can get the key to discriminate the difference of the sound stimuli "021" and "023." In other words, the auditory system can differentiate the AM phase difference of 90 degrees from that of 270 degrees. However, when $f_{\text {mod }}$ is faster than $8 \mathrm{~Hz}$, the auditory system cannot exactly follow each amplitude modulation and can only get the information of envelope correlation. In this case it is impossible for the auditory system to differentiate AM phase difference of 90 degrees from that of 270 degrees. There is no timbre difference between " 021 " and "023."

When the amplitude modulation is faster than the temporal resolving power, the auditory system cannot discriminate the phase difference of AM if the envelope correlation is the same. In contrast, the auditory system can discriminate the phase difference of AM if the envelope correlation is different in spite of the modulation frequency.

\section{CONCLUSION}

The timbre of compound tones consisting of three amplitude modulated waves was examined by psychoacoustical experiments using a similarity judgement. The obtained data were analyzed by an MDS method.

The two dimensional MDS solutions of the present experiments are interpreted by the regression equations as a function of the envelope correlation coefficients between the adjacent AM waves. The third dimension at $f_{\text {mod }}=8 \mathrm{~Hz}$ shows the effect of the envelope correlation between the fundamental and the third AM waves. The third dimension at $f_{\text {mod }}=4 \mathrm{~Hz}$ shows the fine structure of amplitude envelopes of each AM wave. However, the weights of the third dimensions are generally small and show significant individual differences. The third dimensions do not seem to represent an important quality.

Consequently, at least, it can be indicated that the envelope correlation between adjacent AM waves is the determinant factor of timbre of the compound tones consisting of three amplitude modulated waves.
There are several studies showing that the auditory system has a function to detect the amplitude envelope correlation between the output signals of the different auditory filters (Richards, 1987, 1988; Yost and Sheft, 1989; Green et al., 1990; Moore and Emmerich, 1990). The present study shows how this function contributes to perception of timbre.

The sound stimuli of the present experiments,

$$
\begin{aligned}
& \sum_{i=1}^{3}\left[1+\cos \left(2 \pi f_{\mathrm{mod}} t+\theta_{i}\right)\right] \cos \left(2 \pi i f_{\mathrm{c}} t\right), \\
= & \sum_{i=1}^{3}\left\{(1 / 2) \cos \left[2 \pi\left(f_{\mathrm{c}}-f_{\mathrm{mod}}\right) t-\theta_{i}\right]\right. \\
& +\cos \left(2 \pi i f_{\mathrm{c}} t\right) \\
& \left.+(1 / 2) \cos \left[2 \pi\left(f_{\mathrm{c}}+f_{\mathrm{mod}}\right) t+\theta_{i}\right]\right\} .
\end{aligned}
$$

This equation shows that phase differences among the modulation waves can be represented as phase differences of the sinusoidal components. Then, the results of this study can be interpreted as a phase effect on timbre.

The phase effect on timbre is often indicated to be due to the difference in amplitude envelope. A typical example is the perceived differences between QFM (quasi-frequency-modulated) and AM tones (Goldstein, 1966). Both QFM and AM tones consist of three components. The difference between them is a 90-degree phase difference of carrier components. The peak factor (difference between maximum and average) of amplitude envelope of AM tones is greater than that of QFM tones. If the bandwidth of the three components exceeds the bandwidth of the auditory filter, the difference between QFM and AM tones cannot be discriminated. The perceived difference between QFM and AM tones shows the phase effect of the components within a bandwidth of auditory filter.

The present study shows another kind of phase effect. The phase differences of components of each AM wave affect amplitude envelope correlations between outputs of each auditory filter. The amplitude envelope correlation is suggested to be a determinant factor of timbre. The present study shows the phase effect of the components across auditory filters.

\section{ACKNOWLEDGEMENTS}

The author would like to thank Koyo Nagoya for his kind assistance in the present work. 


\section{S. IWAMIYA: THE EFFECT OF AMPLITUDE ENVELOPES ON TIMBRE}

\section{REFERENCES}

Arabie, P., Carroll, J. D., and DeSarbo, W. S. (1987). Three-way Scaling and Clustering (Sage Publications, Inc., Newbury Park).

Fletcher, H. and Sanders, L. C. (1967). "Quality of violin vibrato tones," J. Acoust. Soc. Am. 41, 15341544.

Goldstein, J. L. (1966). "Auditory spectral filtering and monaural phase perception," J. Acoust. Soc. Am. 41, 458-479 (1966).

Green, D. M., Richards, A. M., and Onsan, Z. A. (1990). Sensitivity to envelope coherence," J. Acoust. Soc. Am. 87, 323-329.

Moore, B. C. J. and Emmerich, D. S. (1990). "Monaural envelope correlation perception, revised: Effects of bandwidth, frequency separation, duration, and relative level of the noise bands," J. Acoust. Soc. Am. 87, 2628-2633.

Richards, V. M. (1987). "Monaural envelope correlation perception," J. Acoust. Soc. Am. 82, 1621-1630.

Richards, V. M. (1988). "Components of monaural envelope correlation perception," Hear. Res. 35, 47-58.
Seashore, C. E. (1938). Psychology of Music (McGrawHill, New York), pp. 20, 33-52.

Sundberg, J. (1982). "Perception of singing," in The Psychology of Music, D. Deutsch, Ed. (Academic Press, New York), Chap. 3, pp. 59-98.

Yost, W. A. and Sheft, S. (1989). "Across-critical-band processing of amplitude-modulated tones," J. Acoust. Soc. Am. 85, 848-857.

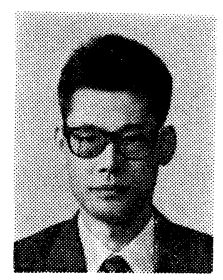

Shin-ichiro Iwamiya was born in Kobe, Japan in 1952. He received his Bachelor degree from Kyushu Institute of Design in 1975, and his Ph. D. from Tohoku University in 1990 . He was a visiting scholar of the University of California, Los Angeles in 1993. He received the 33rd Sato Paper Award of the Acoustical Society of Japan. He is an Associate Professor of Department of Acoustic Design, Kyushu Institute of Design. His research interests include psychoacoustics, psychomusicology, and acoustic ecology. 